\title{
PC to PC Transfer of Text, Images Using Visible Light Communication (VLC)
}

\author{
Kosuri Siva Satyanarayana Raju, Velisetti Mohana Venkata Sai Deekshith
}

\author{
Department of ECE, VIT University, Vellore, Tamilnadu
}

\begin{abstract}
Light Emitting Diodes are assuming a noteworthy part in numerous regions of our everyday life. Aside from transmitting light, these LED's can be used for data transmission utilizing Visible Light Communication (VLC). In this paper, We propose a complete model to transfer text, images from one device to another using Visible light communication, in which transmitter transmits the encrypted data through visible light and the receiver at the receiving end identifies the transmitted data and decrypts it to retrieve the data sent.
\end{abstract}

Keywords- LCD, LED, MAX232, UART, Visible light communication (VLC).

\section{INTRODUCTION}

With the expanding requests for the wireless services, the present radio frequency (RF) based solutions, for example, Wi-Fi, computerized digital TV etc., need to manage some genuine ghastly congestion issues, particularly in swarmed places like inns, hostels or thick private structures. In this context, visible light communications (VLC) which utilize the illuminating light-emitting diode (LED) for transmission, offers a huge and unlicensed bandwidth to cope with crowded radio spectrum for highly-localized communication systems. Visible Light Communication is a kind of correspondence in which air goes about as medium of transmission, visible light in the vicinity of 400 and 800 $\mathrm{THz} \quad(780-375 \mathrm{~nm})$ acts as signal transmitter and photodiode is utilized as signal receiver. Besides, the VLC technology has many other attractive features, such as unlicensed bandwidth, radiation free communication, highcapacity, etc., and hence is considered as an appealing alternative of RF technology for indoor coverage. However Visible Light Communication acts as a backbone to provide access to unused bandwidth. The conventional way of installing VLC is connecting the LED lamps to the modem via network cables, which requires large modification of the indoor layout and is not cost-effective. The combination of VLC and power line communications (PLC) comes into play from the observation that all the LED lamps are connected to the power line and the power line can naturally act as the backbone for VLC while powering the LED lamp. In this way, it will save the additional cables during broadcasting a message to several receivers and it is easier to be installed. Ultraviolet rays and Gamma rays can't be utilized because of the fact that they could be unsafe for people. X-rays have comparative issues on a few sections of the body. Infrared rays can't be utilized for eye well-being and must be utilized with low power. Apart from all these rays only visible rays doesn't create radiation and accomplishes higher security than conventional systems since light doesn't pass through walls of the room.
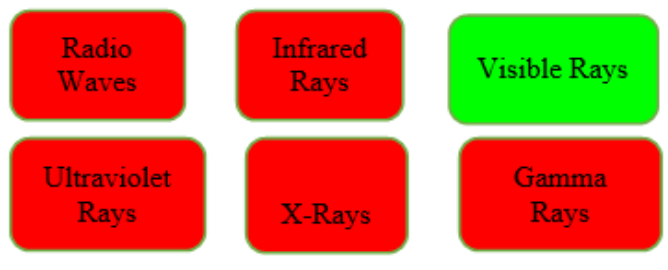

Fig. 1: Regions of electromagnetic spectrum.

LED's plays a major role in this type of radiation less communication. LED's can be able to switch-on and switch-off at very high speeds (operating at less than $1 \mu$ s). So, these changes can't visible to human eyes. With the assistance of high working velocities, LED's can transmit binary information. At the point when LED is "ON", it will be considered as "binary bit 1 ". At the point when LED is "OFF", it will be considered as "binary bit $0 "$ ". Because of the higher flickering speed of LED's one can accomplish higher information rates.

\section{BLOCK DIAGRAM}

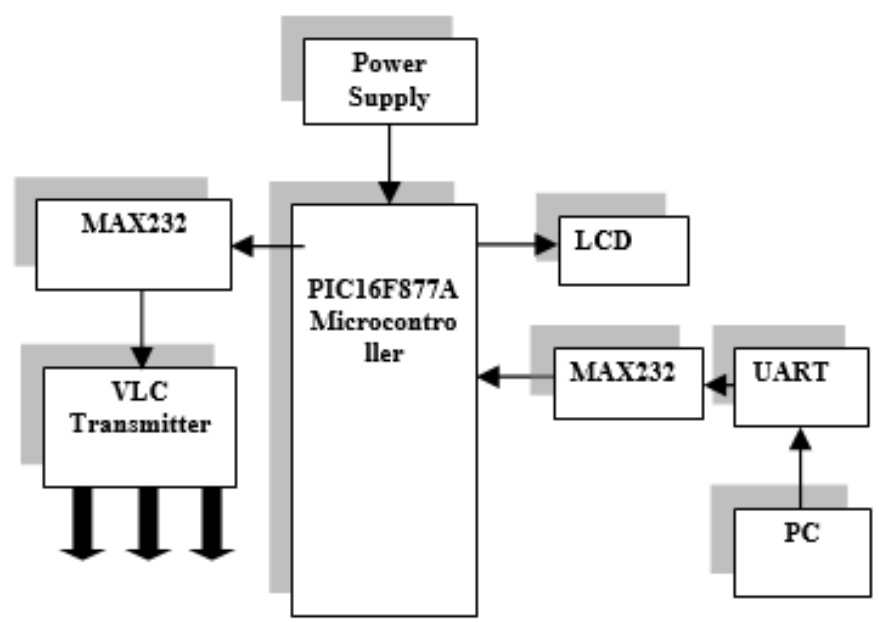

Fig. 2: Block diagram of transmitter section. 


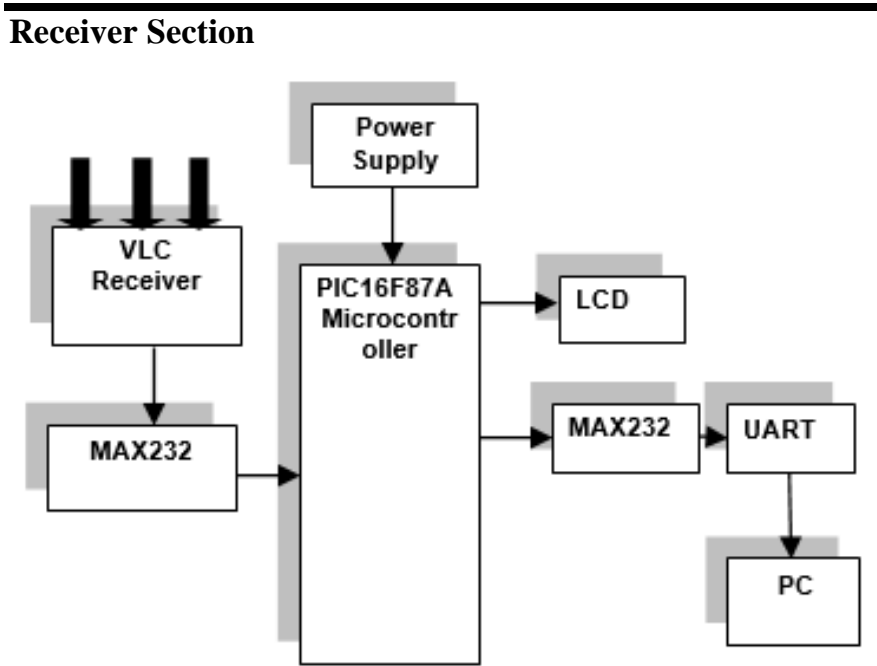

Fig. 3: Block diagram of receiver section.

In this proposed system we are tend to work with PIC16F877A microcontroller. In this project we are transmitting the data from one place to another with the help of VLC transceiver. This can be done through $\max 232$ and UART, $\max 232$ is used to convert Rs 232 into TTL and the data from the pc are transmitting via UART to the controller and transmitted data can be received in the receiver section. The current status will be seen in LCD. Finally, the status is updated in the PC.

\section{CIRCUIT DIAGRAM}

3.1 Transmitter side:

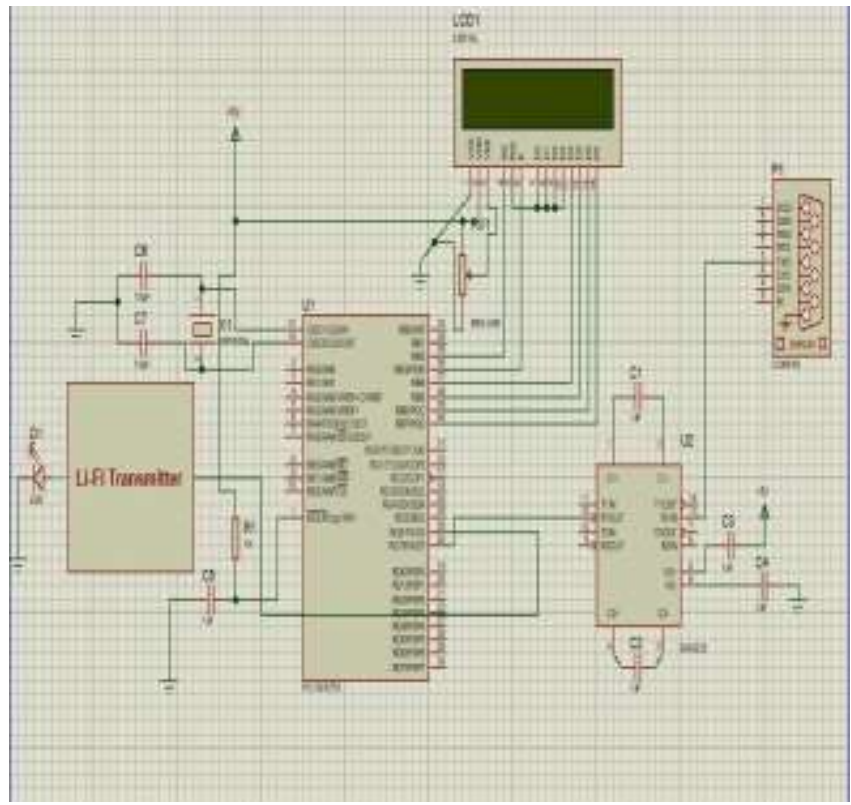

Fig. 4: Transmitter side circuit diagram.

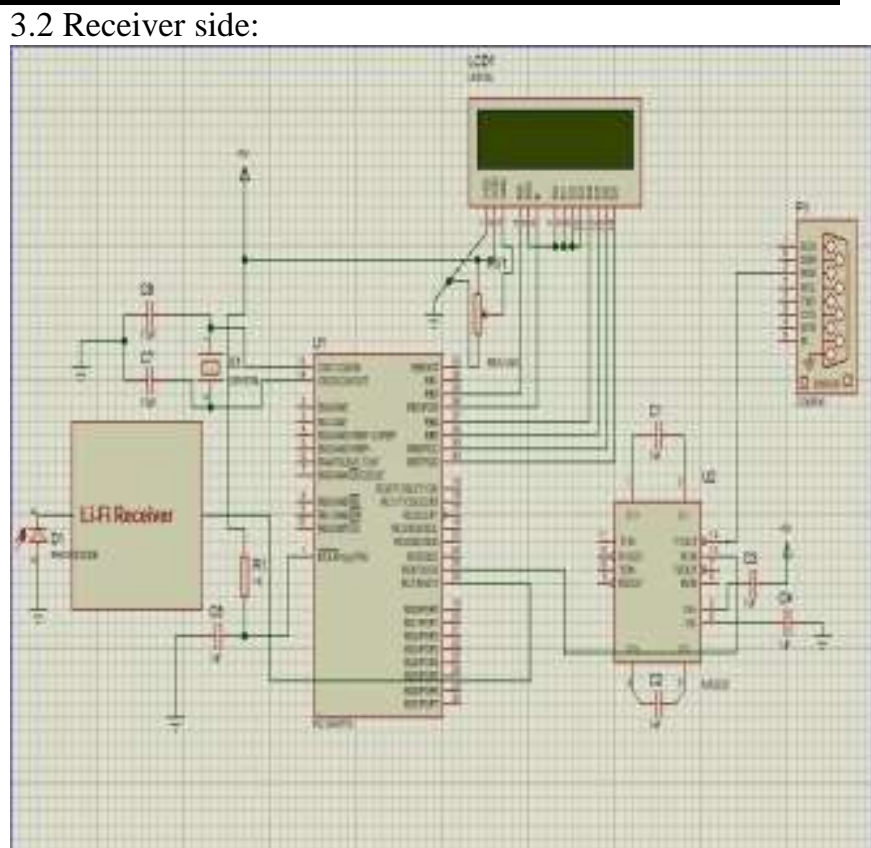

Fig. 5: Receiver side circuit diagram.

\section{WORKING PRINCIPLE}

\subsection{AT TRANSMITTER:}

The transmission pc accepts the data and converts data into its respective ASCII form. This data is transmitted via UART to MAX232. The output of UART provides serial data. MAX232 is used to synchronize the voltage levels between microcontroller and RS232 logic levels from pc. Max232 sends serial data to microcontroller where serial data is framed and fed to VLC transmitter end circuit. This VLC transmitter generally consists of 4 components namely, bulb, LED driver circuit, PCB board, Enclosure. A modulator is used to combine the data stream with carrier signal by using AND gate. The LED driver circuit powers the LED at very high speeds (response time is less than $1 \mu \mathrm{s})$ according to modulated signal.

\subsection{AT RECEIVER:}

The transmitted light is made to illuminate on receiver which is $\mathrm{Si}$ photodiode. The information received by photodiode is converted into electrical pulses. A transimpedance amplifier is used to convert electrical pulses to voltages. These voltages are then fed to comparator to deal with distortion in the signal. Then the signal is fed to digital signal modulator which separates data signal and carrier signals. Thus obtained demodulated data signal is given to microcontroller for reframing and then send it to receiver pc serial communication port through MAX232 and UART. The data that received can be shown in receiver side pc hyperterminal window. 


\section{LITERATURE REVIEW}

Now-a-days, majority of us are familiar with Wi-Fi (Wireless Fidelity), which generally uses $2.4,5 \mathrm{GHz}$ radio frequencies to transmit data wirelessly. But, these radio waves are harmful for living beings. So, the best alternative for this problem is Visible Light Communication (VLC), where LED lights are used to transfer the data wirelessly. VLC is recently referred as $\mathrm{Li}$ Fi. It is a term often used to describe high speed VLC in application scenarios where Wi-Fi might also be used. The term $\mathrm{Li}-\mathrm{Fi}$ is similar to Wi-Fi with the exception that light rather than radio is used for transmission. Professor Harald Haas, from the University of Edinburgh in the UK, is widely recognized as the original founder of $\mathrm{Li}-\mathrm{Fi}$.

By the end of AUGUST 2013, data rates of over 1.6 GBPS were achieved using Li-Fi (light fidelity).

In APRIL 2014, the Russian company Stins Coman has announced the development of a $\mathrm{Li}-\mathrm{Fi}$ wireless local network called Beam Caster. They achieved data rates of 1.25 GBPS

With $\mathrm{Li}-\mathrm{Fi}$, we can able to communicate under water, gives more security compares to $\mathrm{Wi}-\mathrm{Fi}$ as light cannot pass through wall of the room.

\section{HARDWARE CIRCUIT PROTOTYPE}

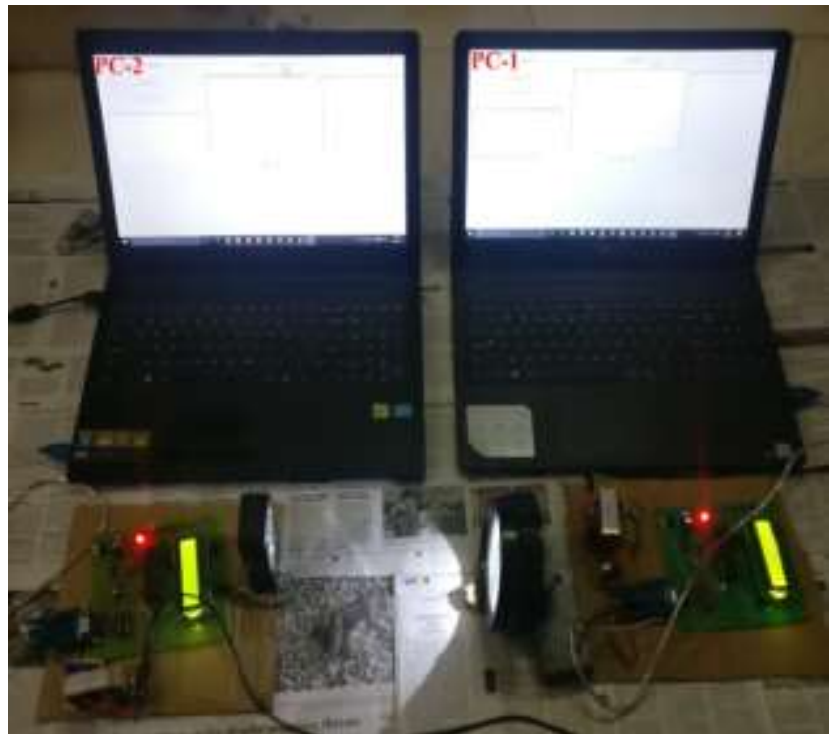

Fig. 6: Complete hardware arrangement.

Figure 6 shows our complete hardware arrangement with which data (Text, Images) can be transferred. In this model, we tried our equipment with virtual products like HyperTerminal, Li-Fi photograph exchange program to transfer text and images from PC-1 to PC-2. The Software tools are initialized with a baud rate of $9600 \mathrm{kbps}$ and all others are set to their default values.

\subsection{Text transfer:}

\section{RESULTS}

In the first place we sent text from PC-1 to PC-2 then we continued with picture exchange. Figure 7 demonstrates the exchange of text from PC-1 to PC-2.

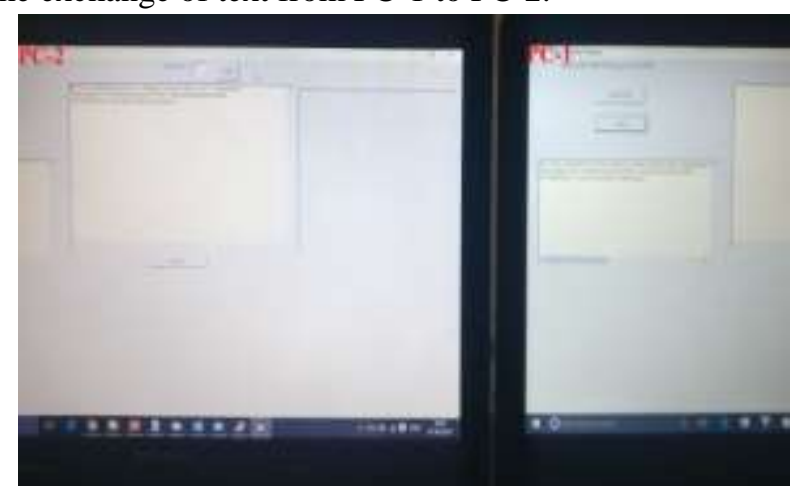

Fig. 7: Transfer of text via Visible Light Communication (VLC).

\subsection{Image transfer:}

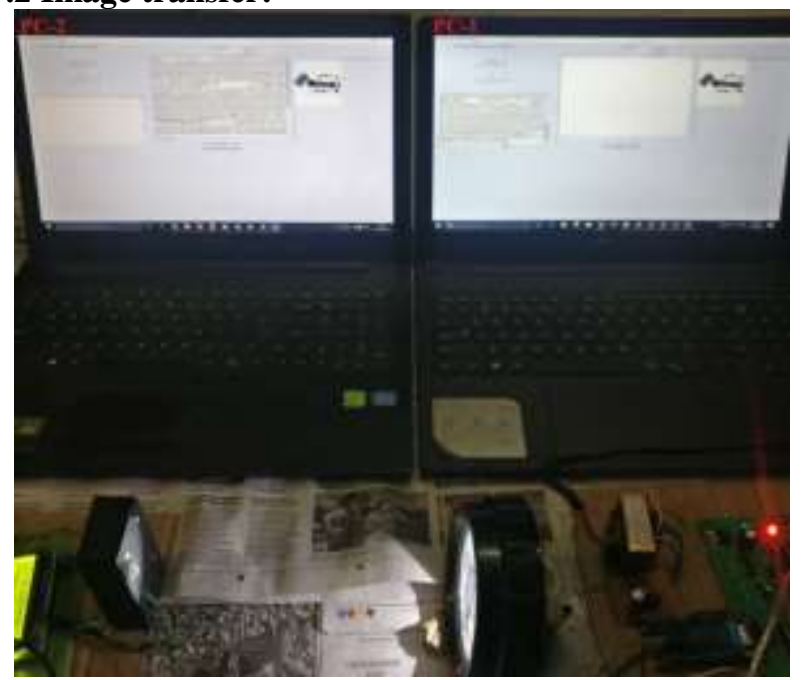

Fig. 8: Transfer of image via Visible Light Communication (VLC).

Thus our project was successful in sending the text as well as image from PC-1 to PC-2 with text accuracy of $100 \%$ and image exactness of $99 \%$.

\section{OBSERVATIONS}

The experiment was tested successfully and data was sent from one pc to another pc using VLC, and the observations are discussed below,

1. The maximum distance between transmitter and receiver is 4 meters to achieve successful transmission.

2. Transmission distance can be increased by using high capacity LED's.

3. Always line of sight must be present between transmitter and receiver. 
4. Baud rate can be manually varied inside software code and hyperterminal software. In this paper, we achieved a baud rate of $9600 \mathrm{kbps}$.

\section{CONCLUSION}

A pc to pc transfer of text, images utilizing Visible Light Communication (VLC) has been presented. The potential outcomes are immeasurable and can be investigated facilitate. In the event that this innovation can be put into down to earth utilize, each bulb can be utilized as a light source alongside VLC based Wi-Fi hotspots.

\section{REFERENCES}

[1] Wasiu O. Popoola; Evangelos Pikasis "On visible light communication and quality of light emitted from illumination LEDs. IEEE photonics society summer topical meeting series. Year: 2016.

[2] http://visiblelightcomm.com/what-is-visible-lightcommunication-vlc/

[3] Watts, Michael (31 January 2012). "Meet Li-Fi, the LED-based alternative to household Wi-Fi". Wired Magazine.

[4] Sathiya.T, Prof.E.Divya, Prof.S.Raja, "Visible Light Communication for Wireless Data Transmission", International Journal of Innovative Research In Electrical, Electronics, Instrumentation and Control Engineering" Vol. 2, Issue No. 2, February 2014.

[5] K. Sindhubala and B. Vijayalakshmi, "Design and Implementation of Visible Light Communication System In Indoor Environment", ARPN Journal of Engineering and Applied Sciences", VOL. 10, NO. 7, APRIL 2015.

[6] K. Cui, G. Chen, Z. Xu, and R. D. Roberts, "Lineof sight visible light communication system design and demonstration," Proc. of 7th IEEE, IET International Symposium on Communication Systems, Networks and Digital Signal Processing. Year: 2010.

[7] Ekta, Ranjeet Kaur Light -Fidelity (LI-FI)-A Comprehensive Studyll International Journal of Computer Science and Mobile Computing Vol. 3, Issue. 4, April 2014, pg.475 - 481 ISSN 2320088X.

[8] Visible Light Communications: challenges and Possibilities.

[9] Dominic C. O'Brien, Lubin Zeng1, Hoa Le-Minh, Grahame Faulkner, Joachim W. Walewski, Sebastian Randel,University of Oxford (UK); Siemens AG, Corporate Technology, Information and Communications, Munich.

[10] Shaista Tarannum, "Data Transmission Through Smart Illumination via "Visible Light
Communication Technology", IJTRA, Volume 4, Year: 2016. 